\title{
An unusual presentation of herpes simplex esophagitis: a nonhealing "peptic" ulcer
}

A 58-year-old man presented with progressive dysphagia, odynophagia, and heartburn. Past history included diabetes mellitus and asthma. Current medications included oral and inhaled corticosteroids. Examination was unremarkable. Blood investigations were normal. Barium swallow demonstrated reflux esophagitis. Endoscopy revealed circumferential ulceration and a hiatus hernia. Biopsy demonstrated an esophageal ulcer and esophagitis with no evidence of malignancy. Despite high-dose treatment with proton pump inhibitors (PPI), ulceration persisted. CT scan and endoscopic ultrasonography showed a diffuse thick-walled proximal esophagus but no typical appearances of carcinoma. Regular endoscopies over 2 years consistently revealed a suspicious circumferential ulcer from 22 to $25 \mathrm{~cm}$ ( $\bullet$ Fig. 1).

Repeated biopsies suggested esophagitis with no evidence of malignancy, infection, or Crohn's disease. After 2 years, biopsies indicated herpes simplex. This was confirmed by viral immunoglobulin levels and treated with aciclovir. Immunodeficiency was excluded. Follow-up revealed the patient to be asymptomatic. Endoscopy demonstrated a completely healed mucosa ( $\bullet$ Fig. 2).

Nonhealing gastroesophageal ulcers are usually malignant. Most benign ulcers are peptic [1] and $85 \%-96 \%$ respond to high-dose PPI within 8 weeks [1]. Crohn's disease is a rarer cause.

Herpes ulcers are rare but have been documented in immunocompromised and immunocompetent patients [2,3]. Herpes simplex esophagitis is normally self-limiting and associated with orolabial lesions [2]. Typical findings include superficial stellate ulcers in the mid esophagus [3]. Advanced herpes simplex esophagitis leads to plaques, “cobblestones”, and an ulcerative appearance. Earlier stages have rounded vesicles which slough into sharp demarcated ulcers which may coalesce [3]. Herpes esophagitis is best diagnosed

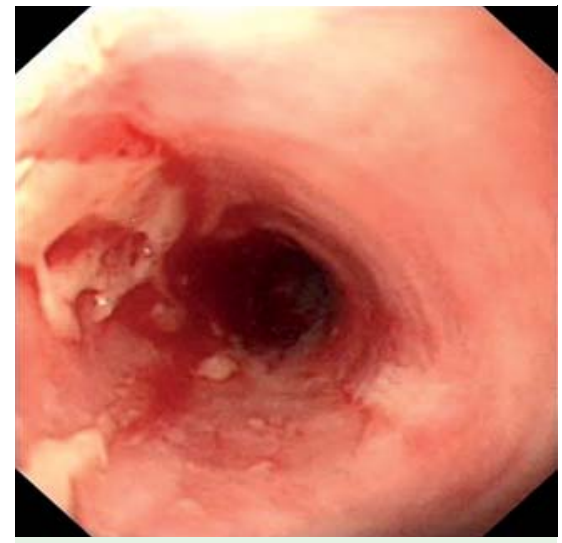

Fig. 1 Herpes esophageal ulceration.

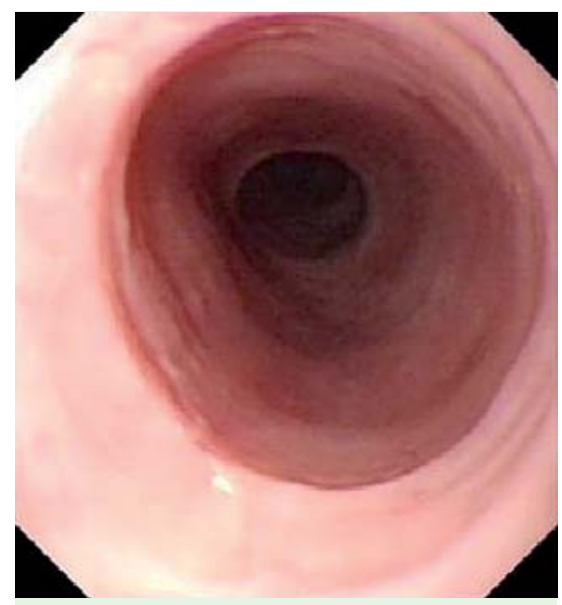

Fig. 2 Completely healed esophageal ulcer after aciclovir therapy.

from viral culture or immunostaining of a biopsy from the ulcer edge. Patients require antiviral therapy [2].

This is an unusual presentation of a herpes simplex esophageal ulcer, which highlights the importance of repeated endoscopies and biopsies as features are not always identified initially. It is important to remember herpes simplex as a cause of a nonhealing ulcer or the chance for curative therapy may be missed.

Endoscopy_UCTN_Code_CCL_1AB_2AC_3AZ

\section{A. G. N. Robertson, L. J. Dunn,} A. Immanuel, S. M. Griffin

Northern Oesophago-Gastric Cancer Unit, Royal Victoria Infirmary, Newcastle Upon Tyne, UK

\section{References}

1 Fass $R$. Symptom assessment tools for gastroesophageal reflux disease (GERD) treatment. J Clin Gastroenterol 2007; 41: 437 444

2 Baehr PH, McDonald GB. Esophageal infections: risk factors, presentation, diagnosis, and treatment. Gastroenterology 1994; 106: 509-532

3 Ramanathan J, Rammouni M, Baran JJr et al. Herpes simplex virus esophagitis in the immunocompetent host: an overview. Am J Gastroenterol 2000; 95: 2171 - 2176

\section{Bibliography}

DOI $10.1055 / s-0029-1214687$

Endoscopy 2009; 41: E213

(c) Georg Thieme Verlag KG Stuttgart · New York . ISSN 0013-726X

\section{Corresponding author}

\section{S. Michael Griffin, MD, FRCS}

Professor of Gastrointestinal Surgery

Northern Oesophago-Gastric Cancer Unit

Royal Victoria Infirmary

Newcastle upon Tyne

NE1 4LP

UK

Fax: +44-191-2820237

Michael.griffin@nuth.nhs.uk 